NT@UW-01-015

\title{
Ioffe Time in Double Logarithmic Approximation
}

\author{
Yuri V. Kovchegov ${ }^{1}$ and Mark Strikman ${ }^{2}$ \\ ${ }^{1}$ Department of Physics, University of Washington, Box 351560 \\ Seattle, WA 98195 \\ ${ }^{2}$ Department of Physics, Pennsylvania State University \\ University Park, PA 16802
}

\begin{abstract}
We analyze the light cone (Ioffe) time structure of the gluon distribution function in the double logarithmic approximation. We show that due to QCD evolution Ioffe equation is modified. The characteristic light cone time of the gluons does not increase as fast with increasing energy (decreasing Bjorken $x$ ) as predicted by the parton distributions exhibiting Bjorken scaling due to the increase of the transverse momenta of the gluons in the DGLAP ladder.
\end{abstract}

\section{Introduction}

It was first observed in Quantum Electrodynamics [1] that photon emission in the scattering process of electrons propagating through a medium occurs over distances increasing with energies. It their seminal paper [2] Gribov, Ioffe and Pomeranchuk have demonstrated that at high energies large longitudinal distances, which are now usually referred to as coherent length distances, $l_{c}$, become important in the virtual photon-nucleon scattering when considered in the rest frame of the target. (One can also formulate this statement in 
the frame independent language by using light-cone time along the reaction axis.) In the following years Ioffe [3] built an explicit connection between the deep inelastic scattering amplitude and the space-time representation of the correlator of the electromagnetic currents. He analyzed the first deep inelastic data from SLAC assuming the exact Bjorken scaling and demonstrated that the longitudinal distances in the the Bjorken limit are growing as

$$
l_{c} \propto \frac{1}{m_{N} x},
$$

where $m_{N}$ is the target nucleon's mass. A natural implication of the increase of longitudinal distances was the parallel observation by Gribov [4 that large shadowing effects should be present in the deep inelastic scattering off nuclei at small enough $x$ when $l_{c} \gg 2 R_{A}$, leading to the saturation behavior of $F_{2 A} \propto A^{2 / 3} Q^{2} \ln (1 / x)$.

Qualitatively one can understand the pattern of Eq. (1) by considering the energy denominator for the transition of the virtual photon to some excited Fock state consisting of quarks and gluons with the invariant mass $M^{2}$. Indeed the uncertainty principle tells us that the lifetime of this state in the nucleon rest frame is the inverse of the energy non-conservation in the $\gamma^{*} \rightarrow$ " $M$ " transition and hence $\sim 2 q_{0} /\left(M^{2}+Q^{2}\right)$, which for $M^{2}$ comparable to $Q^{2}$ leads to Eq. (11). Bjorken [5] has build an explicit picture of the space time evolution of the quark-antiquark pair in the aligned jet model of DIS for the transition $\gamma^{*} \rightarrow q \bar{q}$ and demonstrated that even though the pair is produced at zero transverse separation it reaches hadron size scale over the time comparable to the coherent length $l_{c}$ of Eq. (1).

Further studies of $l_{c}$ were performed in [6] in the aligned jet model. It was demonstrated that in this model the average value of $M^{2}$ is $\left\langle M^{2}\right\rangle \approx Q^{2}$, leading to $l_{c}=\frac{1}{2 m_{N} x}$. Similar numbers were obtained in [6] by explicit consideration of the Ioffe representation assuming that the structure function exhibits Bjorken scaling and that the nucleon's structure function behaves as $F_{2 N}(x) \rightarrow$ const in the $x \rightarrow 0$ limit.

More recently in a number of papers the coordinate representation of nucleonic and nuclear correlators of electromagnetic currents was considered using the current parameterizations of parton densities and employing the Bjorken scaling approximation for the Ioffe expressions [7, 8]. The essential distances in the coherent diffractive interactions with nuclei which lead to the leading twist shadowing were investigated in [9] and coherence distances were 
found to be of the order of $\sim 1 / 2 m_{N} x$ for moderate $Q^{2}$ but were decreasing with increasing $Q^{2}$.

The aim of this paper is to investigate how the scaling violation which is especially strong in the region of small $x$ affects previous conclusions for the value of $l_{c}$. Qualitatively we can expect that due to production of intermediate states with a large invariant mass in the ladder-type kinematics the coherence time $l_{c}$ should be substantially reduced. As a first step we will use a double logarithmic approximation which emphasizes the ladder kinematics. In Sect. 2 we will consider the double logarithmic evolution with fixed strong coupling constant and in Sect. 3 we will analyze the case of running coupling constant in the evolution equations.

In Sect. 4 we will study how the new value for $l_{c}$ generated by the double logarithmic evolution affects the onset of non-linear effects such as saturation [1], 10, 11, 12] in a nucleus. To do that we will have to estimate at what energies the coherence length becomes larger than the nuclear diameter $2 R_{A}$. As we will observe the double logarithmic evolution slows down the onset of these phenomena with energy.

We want to analyze the structure of the gluon distribution in a proton in terms of the Ioffe time variable $u=p \cdot x$, where $p_{\mu}$ is the target nucleon's momentum and $x_{\mu}$ is the parton's coordinate. To avoid confusion throughout the paper we will write $z$ for the Bjorken $x$ variable. The gluon distribution function can be represented as a Fourier transform in light cone time $u$ as [13]

$$
z G\left(z, Q^{2}\right)=\int_{-\infty}^{\infty} d u e^{i u z} f(u, \nu)
$$

with $\nu=p \cdot q / m_{N}$ and $Q^{2}=-q^{2}$ the photon's virtuality. Here the function $f(u, \nu)$ in general also depends on the transverse momentum squared $\underline{q}^{2}$. Inverting the Fourier transform of Eq. (2) we write

$$
f(u, \nu)=\int_{0}^{\infty} \frac{d z}{2 \pi} e^{-i u z} z G\left(z, 2 m_{N} \nu z\right)-\text { complex conjugate. }
$$

Therefore, to determine the coherence length $l_{c}$, or, equivalently, the characteristic Ioffe time $u$ of the gluon distribution we have to find the function $f(u, \nu)$ using the inverse Fourier transform of Eq. (3) and extract the typical values of $u$ out of it. 


\section{Ioffe Time Evolution of the Double Log- arithmic Structure Function: Fixed Cou- pling Case}

We begin by considering the gluon distribution function of a hadron in the double logarithmic approximation. The DGLAP equation [14 for the gluon structure function in the double logarithmic approximation is

$$
Q^{2} \frac{\partial}{\partial Q^{2}} G\left(z, Q^{2}\right)=\frac{\alpha_{s}\left(Q^{2}\right)}{2 \pi} \int_{z}^{1} \frac{d z^{\prime}}{z^{\prime}} \gamma_{G G}\left(\frac{z}{z^{\prime}}\right) G\left(z^{\prime}, Q^{2}\right)
$$

where the gluon-gluon splitting function at small $z$ is given by

$$
\gamma_{G G}(z) \approx \frac{2 N_{c}}{z}
$$

To solve Eq. (田) we for simplicity choose the following initial condition at some not very large value of photon's virtuality $Q_{0}^{2}$

$$
G\left(z, Q_{0}^{2}\right)=\delta\left(z-z_{0}\right) .
$$

In this section we will consider the "toy model" case when the strong coupling constant is fixed. Then the solution of Eq. (4) is given by

$$
z G\left(z, Q^{2}\right)=\int \frac{d n}{2 \pi i}\left(\frac{z_{0}}{z}\right)^{n-1}\left(\frac{Q^{2}}{Q_{0}^{2}}\right)^{\frac{\alpha_{s} N_{c}}{\pi} \frac{1}{n-1}},
$$

where the integral over $n$ runs along a straight line parallel to imaginary axis to the right of all the singularities of the integrand. Using Eq. (7) in Eq. (3) we obtain

$$
\begin{gathered}
f(u, \nu)=\int_{0}^{\infty} \frac{d z}{2 \pi} e^{-i u z} \int \frac{d n}{2 \pi i}\left(\frac{z_{0}}{z}\right)^{n-1-\frac{\alpha_{s} N_{c}}{\pi} \frac{1}{n-1}}\left(\frac{\nu}{\nu_{0}}\right)^{\frac{\alpha_{s} N_{c}}{\pi} \frac{1}{n-1}} \\
- \text { complex conjugate. }
\end{gathered}
$$

Performing the $z$ integration in Eq. (8) yields

$$
f(u, \nu)=-\frac{1}{|u|} \int \frac{d n}{2 \pi^{2}} \Gamma\left(2-n+\frac{\alpha_{s} N_{c}}{\pi} \frac{1}{n-1}\right)
$$




$$
\times \sin \left[\frac{\pi}{2}\left(n-\frac{\alpha_{s} N_{c}}{\pi} \frac{1}{n-1}\right)\right]\left(u z_{0}\right)^{n-1-\frac{\alpha_{s} N_{c}}{\pi}} \frac{1}{n-1}\left(\frac{\nu}{\nu_{0}}\right)^{\frac{\alpha_{s} N_{c}}{\pi} \frac{1}{n-1}}
$$

where the integration over $n$ can be approximated by the saddle point method around

$$
n_{0}-1=\sqrt{\frac{\alpha_{s} N_{c}}{\pi} \ln \frac{\nu}{u z_{0} \nu_{0}} \frac{1}{\ln u z_{0}}}
$$

to give

$$
\begin{aligned}
& f(u, \nu)=-i \frac{1}{|u|}\left(\frac{\alpha_{s} N_{c} \pi \ln \frac{\nu}{u z_{0} \nu_{0}}}{\ln ^{3} u z_{0}}\right)^{1 / 4} \\
& \quad \times \exp \left(2 \sqrt{\frac{\alpha_{s} N_{c}}{\pi} \ln \frac{\nu}{u z_{0} \nu_{0}} \ln u z_{0}}\right) .
\end{aligned}
$$

Eq. (11) gives the Fourier image in Ioffe time of the gluon structure function in a proton. Our goal is to determine which values of the variable $u$ dominate in Eq. (2) with the function $f(u, \nu)$ given by Eq. (11). Most of the $z$ and $\nu$ dependence in Eq. (11) is driven by the exponential function in it. To determine the characteristic values of $u$ we have to find which $u$ gives the maximum of the function in the exponent. One can readily see that the maximum is reached at

$$
u_{f c}^{*}=\frac{1}{z_{0}} \sqrt{\frac{\nu}{\nu_{0}}} .
$$

Another way of determining the typical value of $u$ is by finding the median of the integral over $u$ in Eq. (2) [6]. Our numerical estimates showed that the discrepancy between that method and the one presented above is not significant in the double logarithmic approximation and thus Eq. (12) does give a good estimate of the typical $u$ in the gluon distribution function.

One can see that the gluon smearing of Eq. (12) is not as large as one would naively expect from the Ioffe equation where in the Bjorken scaling limit there assumed to be no $\nu$ dependence in the integrand of Eq. (2). There the spread of partons is limited only by the Fourier exponent in Eq. (2) yielding the maximum possible value of $u$ to be

$$
u_{\text {max }}^{*}=\frac{1}{z}
$$


which translates itself into the well-known coherence length of the gluon in the proton's rest frame $l_{c o h}=1 / 2 m_{N} z$. Recalling that $\nu=Q^{2} / 2 m_{N} z$ one can see that the time in Eq. (12) grows much slower with decreasing $z$ than the time given by Eq. (13).

To understand what happens it is instructive to rewrite Eq. (12) in terms of the $x_{-}$coordinate and $q_{-}$momentum of the last gluon in the ladder. Since we are working in the infinite momentum frame where $p_{\mu}=\left(p_{+}, 0, \underline{0}\right)$ we write $u=p \cdot x=p_{+} x_{-}$and $\nu=p \cdot q / m_{N}=p_{+} q_{-} / m_{N}$ which leads us to the following expression for the light cone lifetime of the last gluon in the ladder

$$
x_{f c-}^{*}=\frac{2 q_{-}}{\sqrt{Q_{0}^{2} z_{0} 2 p_{+} q_{-}}} \approx \frac{2 q_{-}}{\sqrt{Q_{0}^{2} z_{0} s}}
$$

where we introduced the center of mass energy $s=(p+q)^{2} \approx 2 p_{+} q_{-}$. On the other hand in a frame where several evolution rungs start in the probe [12 we can write down the light cone lifetime of a (longitudinally) soft gluon as

$$
x_{-}^{*}=\frac{2 q_{-}}{\underline{q}^{2}} .
$$

To estimate the typical $\underline{q}^{2}$ of a gluon in the DLA DGLAP evolution taken at a given fixed value of $q_{-}$(or, equivalently, $s$ ) we first note that in the saddle point approximation Eq. (7) leads to

$$
z G\left(z, Q^{2}\right) \propto \exp \left(2 \sqrt{\frac{\alpha_{s} N_{c}}{\pi} \ln \frac{Q^{2}}{Q_{0}^{2}} \ln \frac{z_{0}}{z}}\right) .
$$

Substituting $Q^{2} \approx \underline{q}^{2}$ and $z \approx \underline{q}^{2} / 2 p_{+} q_{-}$we obtain the typical transverse momentum squared of the gluons

$$
\left\langle\underline{q}^{2}\right\rangle_{f c} \approx \sqrt{Q_{0}^{2} z_{0} 2 p_{+} q_{-}} \approx \sqrt{Q_{0}^{2} z_{0} s}
$$

which, after being substituted into Eq. (15) gives us Eq. (14). Now the difference between Ioffe times in the distribution function exhibiting Bjorken scaling and the double logarithmic gluon distribution becomes apparent. As we increase the center of mass energy $s$ by increasing $q_{-}$the typical transverse momentum of the distribution function without any QCD evolution would 
remain constant, while in the case of double logarithmic evolution it would increase with energy (see Eq. (17)) leading to shorter light cone lifetimes in this latter case. The diffusion of the typical transverse momentum with energy is so fast in our Eq. (17) because in this section we considered the toy model of fixed coupling DLA DGLAP equation.

\section{Ioffe Time Evolution of the Double Loga- rithmic Structure Function: Running Cou- pling Case}

Let us repeat the calculation of the previous chapter for the running coupling case. The running coupling constant in Eq. (4) will be taken below at the one-loop level

$$
\alpha_{s}\left(Q^{2}\right)=\frac{1}{b \ln Q^{2} / \Lambda^{2}}
$$

with the beta function $b=\frac{11 N_{c}-2 N_{f}}{12 \pi}$.

The solution of the double logarithmic DGLAP equation for gluon distribution function (4) with the splitting function of Eq. (5) and the initial conditions given by Eq. (6) is

$$
z G\left(z, Q^{2}\right)=\int \frac{d n}{2 \pi i}\left(\frac{z_{0}}{z}\right)^{n-1}\left(\frac{\ln \left(Q^{2} / \Lambda^{2}\right)}{\ln \left(Q_{0}^{2} / \Lambda^{2}\right)}\right)^{\frac{N_{c}}{\pi b(n-1)}}
$$

where, just like in Eq. (7) the integral over $n$ runs along a straight line parallel to imaginary axis to the right of all the singularities of the integrand. Substituting Eq. (19) into Eq. (3) we write for the Ioffe time representation of the gluon structure function [3]

$$
\begin{aligned}
f(u, \nu)=\int_{0}^{\infty} \frac{d z}{2 \pi} e^{-i u z} & \int \frac{d n}{2 \pi i}\left(\frac{z_{0}}{z}\right)^{n-1}\left(\frac{\ln \left(2 \nu m_{N} z / \Lambda^{2}\right)}{\ln \left(2 \nu_{0} m_{N} z_{0} / \Lambda^{2}\right)}\right)^{\frac{N_{c}}{\pi b(n-1)}}- \\
& - \text { complex conjugate. }
\end{aligned}
$$


Integration over $z$ in Eq. (20) is rather complicated but can be simplified if with the leading logarithmic accuracy we substitute $z$ by $1 / u$ in the ratio of the logarithms in it. The rest of the integral can be done easily yielding

$$
\begin{gathered}
f(u, \nu)=-\frac{1}{|u|} \int \frac{d n}{2 \pi^{2}} \Gamma(2-n) \sin \left(\frac{\pi n}{2}\right) \\
\times\left(u z_{0}\right)^{n-1}\left(\frac{\ln \left(2 \nu m_{N} / u \Lambda^{2}\right)}{\ln \left(2 \nu_{0} m_{N} z_{0} / \Lambda^{2}\right)}\right)^{\frac{N_{c}}{\pi b(n-1)}} .
\end{gathered}
$$

The expression in Eq. (21) can be evaluated by the saddle point method. The position of the saddle point is given by

$$
n_{0}-1=\sqrt{\frac{N_{c}}{\pi b} \ln \left(\frac{\ln \left(2 \nu m_{N} / u \Lambda^{2}\right)}{\ln \left(2 \nu_{0} m_{N} z_{0} / \Lambda^{2}\right)}\right) \frac{1}{\ln \left(u z_{0}\right)}}
$$

and Eq. (21) becomes

$$
\begin{aligned}
& f(u, \nu)=-i \frac{1}{2 \pi^{2}|u|}\left[\frac{\frac{N_{c} \pi}{b} \ln \left(\frac{\ln \left(2 \nu m_{N} / u \Lambda^{2}\right)}{\ln \left(2 \nu_{0} m_{N} z_{0} / \Lambda^{2}\right)}\right)}{\ln ^{3}\left(u z_{0}\right)}\right]^{1 / 4} \\
& \times \exp \left(2 \sqrt{\frac{N_{c}}{\pi b} \ln \left(\frac{\ln \left(2 \nu m_{N} / u \Lambda^{2}\right)}{\ln \left(2 \nu_{0} m_{N} z_{0} / \Lambda^{2}\right)}\right) \ln \left(u z_{0}\right)}\right) .
\end{aligned}
$$

Eq. (23) yields the distribution in light cone Ioffe time of the gluon structure function in a proton. Similarly to the previous section in order to find the characteristic value of $u$ we need to find the maximum of the function in the power of the exponent in Eq. (23). Defining $L=\ln \left(2 \nu m_{N} z_{0} / \Lambda^{2}\right)$ and $L_{0}=\ln \left(2 \nu_{0} m_{N} z_{0} / \Lambda^{2}\right)$ together with $\zeta=\ln \left(u z_{0}\right) / L$ allows us to rewrite the function under the square root in the exponent of Eq. (23) as

$$
h(\zeta)=\zeta L \ln \frac{L(1-\zeta)}{L_{0}} .
$$

For $L \gg L_{0}$ the maximum of Eq. (24) is reached at the value of $\zeta$ that can be very well approximated by the formula

$$
\zeta^{*} \approx 1-\frac{1}{\ln L / L_{0}}
$$


which is found from the usual extremum condition

$$
h^{\prime}\left(\zeta^{*}\right)=\ln \frac{L}{L_{0}}+\ln \left(1-\zeta^{*}\right)-\frac{\zeta^{*}}{1-\zeta^{*}} \approx \ln \frac{L}{L_{0}}-\frac{\zeta^{*}\left(2-\zeta^{*}\right)}{1-\zeta^{*}}=0 .
$$

Eq. (25) can be rewritten in terms of the Ioffe time and $\nu$ as

$$
u^{*} \approx \frac{1}{z_{0}}\left(\frac{2 \nu m_{N} z_{0}}{\Lambda^{2}}\right)^{1-\ln ^{-1}\left(\frac{\ln \left(2 \nu m_{N} z_{0} / \Lambda^{2}\right)}{\ln \left(2 \nu_{0} m_{N} z_{0} / \Lambda^{2}\right)}\right)}
$$

or in terms of $x_{-}$and $q_{-}$as

$$
\begin{aligned}
x_{r c-}^{*}= & \frac{2 q_{-}}{\Lambda^{2}}\left(\frac{2 p_{+} q_{-} z_{0}}{\Lambda^{2}}\right)^{-\ln ^{-1}\left(\frac{\ln \left(2 p_{+} q_{-} z_{0} / \Lambda^{2}\right)}{\ln \left(Q_{0}^{2} / \Lambda^{2}\right)}\right)} \\
& \approx \frac{2 q_{-}}{\Lambda^{2}}\left(\frac{s z_{0}}{\Lambda^{2}}\right)^{-\ln ^{-1}\left(\frac{\ln \left(s z_{0} / \Lambda^{2}\right)}{\ln \left(Q_{0}^{2} / \Lambda^{2}\right)}\right)} .
\end{aligned}
$$

To understand this result we, similarly to the previous section first note that in the saddle point approximation the gluon distribution of Eq. (19) is proportional to

$$
z G\left(z, Q^{2}\right) \propto \exp \left(2 \sqrt{\frac{N_{c}}{\pi b} \ln \left(\frac{\ln \left(Q^{2} / \Lambda^{2}\right)}{\ln \left(Q_{0}^{2} / \Lambda^{2}\right)}\right) \ln \frac{z_{0}}{z}}\right)
$$

with the typical transverse momentum for fixed $q_{-}$given by

$$
\left\langle\underline{q}^{2}\right\rangle_{r c} \approx \Lambda^{2}\left(\frac{2 p_{+} q_{-} z_{0}}{\Lambda^{2}}\right)^{\ln -1\left(\frac{\ln \left(2 p_{+} q_{-} z_{0} / \Lambda^{2}\right)}{\ln \left(Q_{0}^{2} / \Lambda^{2}\right)}\right)} \approx \Lambda^{2}\left(\frac{s z_{0}}{\Lambda^{2}}\right)^{\ln ^{-1}\left(\frac{\ln \left(s z_{0} / \Lambda^{2}\right)}{\ln \left(Q_{0}^{2} / \Lambda^{2}\right)}\right)} .
$$

Substituting Eq. (30) into Eq. (15) readily yields us Eq. (28). Thus again the DGLAP evolution makes transverse momenta in the gluon distribution diffuse towards larger values slowing down the spreading of the gluons in the longitudinal $x_{-}$direction as compared to the standard estimate of Eq. (13). The growth of the transverse momenta with $s$ described by Eq. (30) is slower than any positive power of $s$. Thus it is slower than $\left\langle\underline{q}^{2}\right\rangle_{f c} \sim \sqrt{s}$ of the fixed coupling case (see Eq. (17)). Slower growth of the transverse momentum in the running coupling case leads to longer light cone coherence times for the gluons than in the fixed coupling case, as follows from Eq. (15). Nevertheless the coherence time is still shorter than the maximum limit of Eq. (13). 


\section{Discussion}

To illustrate our results we are going to plot the gluon coherence lengths produced by different evolution scenarios discussed above in the rest frame of the proton as functions of center of mass energy s. By doing so we would also address the question posed in the Introduction: at which values of $z$ does the DGLAP evolved gluon distribution of a nucleon in a nucleus reach the lengths comparable to the nuclear diameter, so that the non-linear effects involving multiple rescatterings and mergers of gluon ladders would begin taking place?

In order to plot the coherence length of the gluons in the rest frame of the target proton or nucleus we use the simple relationship $u=1 / 2 m_{N} l_{\text {coh }}$ and employ Eqs. (14) and (28) for the fixed and running coupling cases correspondingly. We use $\Lambda=0.3 \mathrm{GeV}$ and $z_{0}=0.1$. In order for the diffusion of transverse momenta to start at the same initial value we have to choose different values of the initial virtuality $Q_{0}$ for the fixed and running coupling cases: we use $Q_{0}=4 \mathrm{GeV}$ in Eq. (14) and $Q_{0}=0.8 \mathrm{GeV}$ in Eq. (28). By doing so we make the initial transverse momentum squared of both evolutions equal to $q_{\text {init }}^{2} \approx 20 \mathrm{GeV}^{2}$ at $s=200 \mathrm{GeV}^{2}$.

The plot of $l_{c o h}$ as a function of the center of mass energy $s$ is depicted

in Fig. 1. The medium-thick line corresponds to the fixed coupling DLA DGLAP evolution $\left(l_{f c}^{*}\right)$ while the thick line corresponds to the running coupling case $\left(l_{r c}^{*}\right)$. The top solid line depicts the upper limit on the coherence length given by $l_{c o h}=1 / 2 m_{N} z$, where $z=q_{\text {init }}^{2} / s$ to insure that this length is really maximum possible. Finally the horizontal line corresponds to the diameter of some sample large nucleus taken here to be equal to $2 R=14 \mathrm{fm}$. One can see that due to DGLAP evolution the coherence lengths of gluons do not grow as fast with energy $s$ as predicted by the well-known estimate of $1 / 2 m_{N} z$ (upper line). For the case of running coupling the coherence length is roughly a half of the maximum throughout the whole region of $s$ considered. Appropriately, in the case of a nuclear target, the coherence length of the partons in each individual nucleon reaches the nuclear diameter at higher values of $s$ than expected from Eq. (13). In the fixed coupling case the nuclear diameter is reached at the energies beyond those shown in Fig. 1, at about $s \approx 30000 \mathrm{GeV}^{2}$. In a more realistic case of running coupling DGLAP evolution the crossover happens slightly above the one predicted by Eq. (13), leading to about 30\% higher $s$ required for the gluon's coherence length to 


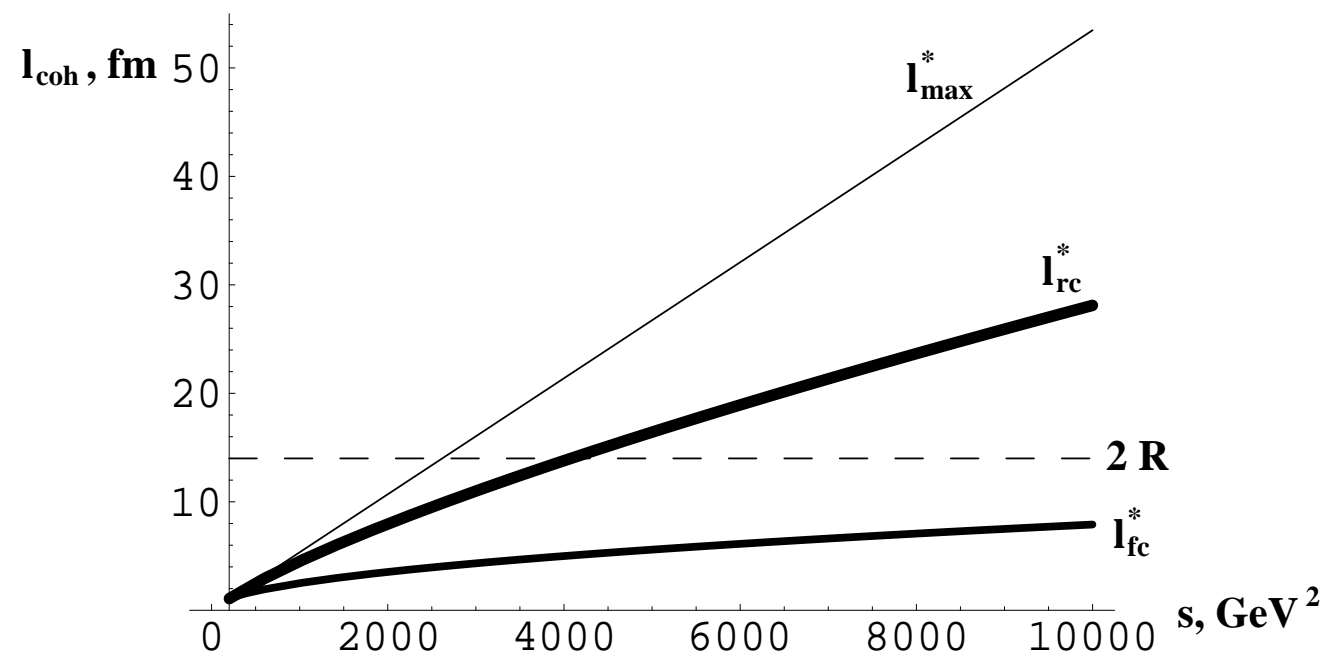

Figure 1: Distances characterizing the spread of gluons in the rest frame of the proton. Horizontal line denotes the diameter of some large nucleus which is taken here to be $14 \mathrm{fm}$. The top solid line corresponds to the upper limit of the Eq. (13), the lower (medium-thick) line corresponds to the fixed coupling DLA DGLAP evolution of Eq. (14) and the middle (thick) line depicts the running coupling evolution of Eq. (28).

become comparable to the nuclear radius (see Fig. 1). To summarize, based on the example of the double logarithmic approximation we conclude that DGLAP evolution towards higher $Q^{2}$ slows down the onset of non-linear effects in the nuclear wave function.

From the picture presented in Fig. 11 one concludes that much smaller than naively expected values of $z$ (for the same large $Q^{2}$ ) are needed in the nuclei for the parton distributions of the individual nucleons to start overlapping making non-linear effect related to nuclear shadowing and parton saturation possible. However one must keep in mind that the spread of the gluon distribution in Fig. 11 is generated by the DLA DGLAP evolution, that is along with going towards smaller values of $z$ the evolution also moves towards larger values of transverse momentum $\underline{q}^{2}$ as could be seen from Eqs. (117) and (30). Going towards smaller $z$ tends to increase light cone times 
and corresponding coherence lengths in the target's rest frame. At the same time increasing $\underline{q}^{2}$ tends to decrease light cone times, moving the evolution away from the saturation region of moderate $\underline{q}^{2} \sim Q_{s}^{2}$. Thus even though DGLAP evolution seems to slow down the growth of the light cone times as functions of $z$ as could be seen from Eqs. (14) and (28), this is entirely due to the fact that DLA DGLAP evolution moves the distributions toward larger $q^{2}$ pushing it away from the saturation region and non-linear effects.

Of course the gluon distribution generated by the double logarithmic evolution equation describes the data well only in a rather narrow kinematic region of small $x$ and large $Q^{2}$. Therefore our analysis of light cone Ioffe time structure of the DLA gluon distribution is also limited to this kinematic region. Further studies involving more realistic parton densities beyond the double log approximation are necessary in order to fully quantify the effect of scaling violations on the light cone structure of distribution functions.

\section{Acknowledgments}

The authors are very much indebted to Lonya Frankfurt for several insightful discussions. M.S. thanks the Department of Energy's Institute for Nuclear Theory at the University of Washington for its hospitality and the Department of Energy for partial support during the time this study started. The work of Yu.K. was supported in part by the U.S. Department of Energy under Grant No. DE-FG03-97ER41014. The research of M.S. was sponsored in part by the U.S. Department of Energy under Grant No. DE-FG02-93ER40771.

\section{References}

[1] L. Landau, I. Pomeranchuk, Dokl. Akad. Nauk Ser. Fiz. 92, 535 (1953).

[2] V.N. Gribov, B.L. Ioffe, I.Ya. Pomeranchuk, Sov. J. Nucl. Phys. 2, 549 (1966); Yad. Fiz. 2, 768 (1965).

[3] B. L. Ioffe, JETP Lett. 9, 163 (1969); 10, 143 (1969); Phys. Lett. 30B, 123 (1969).

[4] V. N. Gribov, Sov. Phys. JETP 30, 709 (1970), Zh. Eksp. Teor. Fiz. 57, 1306 (1969). 
[5] J. D. Bjorken, Electroproduction at Very Small Values of the Scaling Variable, contribution to the Festschrift of Jack Steinberger, K. Kleinknecht and T.D. Lee (editors).

[6] L. Frankfurt and M. Strikman, Phys. Rept. 160, 235 (1988).

[7] G. Piller, M. Vanttinen, L. Mankiewicz, W. Weise, K. Eskola, Nucl. Phys. A663, 328 (2000).

[8] S. Hofmann, L. Mankiewicz, A. Schafer, Z. Phys. A359, 157 (1997); V. Braun, P. Gornicki, L. Mankiewicz, Phys. Rev. D51, 6036 (1995).

[9] L. Frankfurt, V. Guzey and M. Strikman, J. Phys. G 27, R23 (2001).

[10] L. V. Gribov, E. M. Levin, and M. G. Ryskin, Phys. Rep. 100, 1 (1983); A.H. Mueller, J.-W. Qiu, Nucl. Phys. B268, 427 (1986).

[11] L. McLerran and R. Venugopalan, Phys. Rev. D 49, 2233 (1994); 49, 3352 (1994); 50, 2225 (1994); Yu.V. Kovchegov, Phys. Rev. D 54, 5463 (1996); 55, 5445 (1997); J. Jalilian-Marian, A. Kovner, L. McLerran, and H. Weigert, Phys. Rev. D 55,5414 (1997).

[12] I. I. Balitsky, Nucl. Phys. B463, 99 (1996); Yu. V. Kovchegov, Phys. Rev. D 60, 034008 (1999).

[13] B. L. Ioffe, V. A. Khoze, L. N. Lipatov, Hard Processes, vol. 1, Elsevier Science Publishers, (1984).

[14] Yu. L. Dokshitzer, JETP 73, 1216 (1977); V. N. Gribov and L. N. Lipatov, Sov. J. Nucl. Phys. 15, 78 (1972); G. Altarelli and G. Parisi, Nucl. Phys. B126, 298 (1997). 